\title{
Antiplane Problem of Periodically Stacked Parallel Cracks in an Infinite Orthotropic Plate
}

\author{
Long Wu and Pengcheng Du \\ School of Mechanical \& Electronic Engineering, Zhongyuan University of Technology, Zhengzhou 450007, China \\ Correspondence should be addressed to Long Wu; 921246429@qq.com
}

Received 14 May 2014; Accepted 16 July 2014

Academic Editor: Oluwole Daniel Makinde

Copyright (C) $2015 \mathrm{~L}$. Wu and P. Du. This is an open access article distributed under the Creative Commons Attribution License, which permits unrestricted use, distribution, and reproduction in any medium, provided the original work is properly cited.

\begin{abstract}
The antiplane problem of the periodic parallel cracks in an infinite linear elastic orthotropic composite plate is studied in this paper. The antiplane problem is turned into the boundary value problem of partial differential equation. By constructing proper Westergaard stress function and using the periodicity of the hyperbolic function, the antiplane problem of the periodic parallel cracks degenerates into an algebra problem. Using the complex variable function method and the undetermined coefficients method, as well as with the help of boundary conditions, the boundary value problem of partial differential equation can be solved, and the analytic expressions for stress intensity factor, stress, and displacement near the periodical parallel cracks tip are obtained. When the cracks spacing tends to infinity, the antiplane problem of the periodic parallel cracks degenerates into the case of the antiplane problem of a single central crack.
\end{abstract}

\section{Introduction}

Composite materials are a very promising class of structural materials and widely used in many fields. Defects in the composite materials are easier to cause singular stress and cracks. However, periodic crack is the important model to study the problem of multiple cracks. For simplicity, we can consider the agminate cracks as periodic cracks ideally. And the research on periodic cracks problem contributes to making an intensive understanding of failure mechanism of composite materials; therefore, it is very important to study the periodic cracks problem.

Over the past few decades, the antiplane problem of periodic cracks was investigated by many researchers. For example, by using Fourier transforms method, Erdogan, Ozturk, Chen, and Ding [1-4] studied the antiplane problem in functionally graded materials containing a periodic array of collinear cracks. By using Laplace transform and Fourier transform, Wang and Mai [5] analyzed the dynamic antiplane problem of periodic parallel cracks in an infinite functionally graded material, and the stress intensity factors were obtained. By using distributed dislocation method, Pak and Goloubeva [6] studied the antiplane problem in piezoelectric materials containing a periodic array of parallel cracks.
The stress and the electric displacement intensity factors were obtained. By using complex variable function method, Tong et al. [7] studied the antiplane problem in piezoelectric materials containing a doubly periodic cracks of unequal size, and a closed form solution of stress intensity factor was obtained. By using the method of conformal mapping, Hao and $\mathrm{Wu}[8,9]$ considered the antiplane problem on parallel periodical cracks of finite length starting from the interface of two half-planes, and the stress intensity factor was obtained. By using the complex variable function method and the undetermined coefficients method, Lekhnitskii [10] studied the antiplane problem of collinear periodic cracks in an infinite orthotropic fiber reinforcement composite plate, and the analytic expressions for stress intensity factors, stress field, and displacement field of the collinear periodic cracks tip were achieved.

The antiplane problem of the periodic parallel cracks in an infinite linear elastic orthotropic composite plate is studied in this paper. The antiplane problem is turned into the boundary value problem of partial differential equation. By constructing proper Westergaard stress function and using the periodicity of the hyperbolic function, the antiplane problem of the periodic parallel cracks degenerates into an algebra problem. The analytic expressions for stress intensity 
factor, stress, and displacement near the periodical parallel cracks tip are obtained.

\section{Mechanical Model}

As seen in Figure 1, we consider an infinite linear elastic orthotropic composite plate with periodic parallel cracks of mode III. The crack length is $2 a$, the crack spacing is $\omega$, and the antiplane shear force is $\tau$.

The relations between the strain and the stress are as follows [10]:

$$
\begin{array}{cc}
\tau_{y z}=Q_{44} \gamma_{y z}, & \tau_{z x}=Q_{55} \gamma_{z x}, \\
\gamma_{y z}=\frac{\partial w}{\partial y}, & \gamma_{z x}=\frac{\partial w}{\partial x},
\end{array}
$$

where $Q_{44}$ and $Q_{55}$ are the principal directions of the elasticity, $w$ is the displacement, $\gamma_{y z}$ and $\gamma_{z x}$ are the strain, and $\tau_{y z}$ and $\tau_{z x}$ are the stress.

The balancing equation is

$$
\frac{\partial \tau_{z x}}{\partial x}+\frac{\partial \tau_{y z}}{\partial y}=0
$$

Substituting (1) into (2), the governing equation of the antiplane problem can be obtained as follows:

$$
Q_{55} \frac{\partial^{2} w}{\partial x^{2}}+Q_{44} \frac{\partial^{2} w}{\partial y^{2}}=0 .
$$

As can be seen in Figure 1, the boundary conditions of the periodic parallel cracks of mode III are as follows:

$$
\begin{gathered}
y \longrightarrow \infty: \tau_{y z}=\tau, \\
-a<x<a, \quad y=n \omega \quad(n=0, \pm 1, \pm 2, \ldots): \\
\tau_{y z}=0 .
\end{gathered}
$$

An analysis of antiplane problem near periodic parallel cracks tip can be turned to find the solution of the boundary value problem of partial differential equations (3) and (4).

The displacement is

$$
w=U(x+s(y-n \omega)) \quad(n=0, \pm 1, \pm 2, \ldots) .
$$

Substituting (5) into (3), the characteristic is obtained [11]:

$$
Q_{44} s^{2}+Q_{55}=0 .
$$

The solutions of the characteristic equation (6) can be set [11] as

$$
s_{1}=i \sqrt{\frac{Q_{55}}{Q_{44}}}=i \beta \quad s_{2}=-i \beta .
$$

Let

$$
z_{1}=x+s_{1}(y-n \omega)+i n \omega=x_{1}+i y_{1},
$$

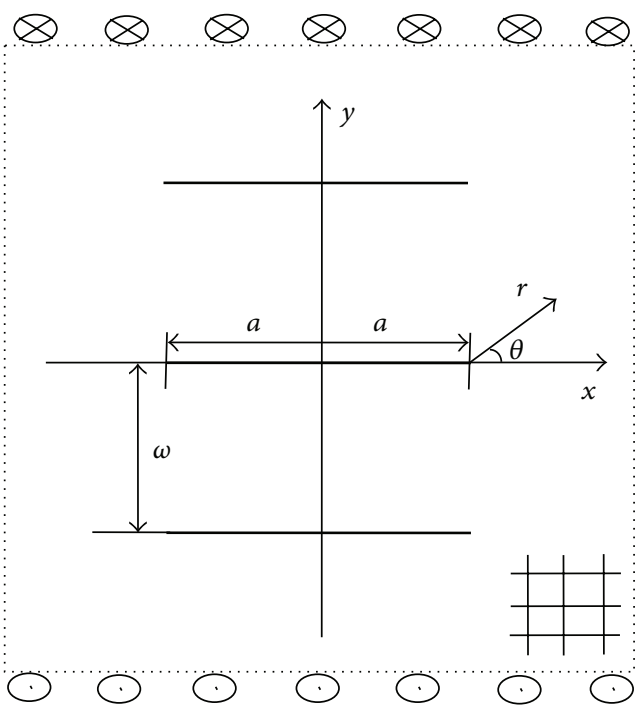

FIGURE 1: Orthotropic plate with periodic parallel cracks of mode III.

where

$$
x_{1}=x, \quad y_{1}=\beta(y-n \omega)+n \omega .
$$

By using formula (8), we can know that the governing equation (3) can be rewritten as a generalized bi-harmonic equation:

$$
\nabla_{1}^{2} U=\left(\frac{\partial^{2}}{\partial x_{1}^{2}}+\frac{\partial^{2}}{\partial y_{1}^{2}}\right) U=0 .
$$

By the theory of complex variable, the solution for partial differential equation (3) may be chosen as [11]

$$
w=U=a_{1} \operatorname{Re}\left(\bar{U}_{1}\right)+b_{1} \operatorname{Im}\left(\bar{U}_{1}\right),
$$

where $a_{1}$ and $b_{1}$ are undetermined real parameters, $U_{1}$ is an analytic function of $z_{1}$, and

$$
\frac{d \bar{U}_{1}}{d z_{1}}=U_{1}=U_{1}\left(z_{1}\right) .
$$

Substituting (11) and (12) into (1), the stress expressions can be written as [11]

$$
\begin{gathered}
\tau_{y z}=Q_{44} \beta\left[-a_{1} \operatorname{Im}\left(U_{1}\right)+b_{1} \operatorname{Re}\left(U_{1}\right)\right], \\
\tau_{z x}=Q_{55}\left[b_{1} \operatorname{Im}\left(U_{1}\right)+a_{1} \operatorname{Re}\left(U_{1}\right)\right] .
\end{gathered}
$$

\section{Westergaard Stress Function}

Considering the boundary value problem of partial differential equations (3) and (4), we can select the Westergaard stress function as follows [12]:

$$
U_{1}\left(z_{1}\right)=\frac{\tau \operatorname{sech}(2 \pi(a+n \omega i) / \omega) \cdot \tanh \left(2 \pi z_{1} / \omega\right)}{\sqrt{\tanh ^{2}\left(2 \pi z_{1} / \omega\right)-\tanh ^{2}(2 \pi(a+n \omega i) / \omega)}},
$$

when $y \rightarrow+\infty: U_{1}\left(z_{1}\right)=\tau$. 


$$
\begin{aligned}
& \text { When }-a<x<a, y=n \omega(n=0, \pm 1, \pm 2, \ldots), \\
& U_{1}\left(z_{1}\right)=\frac{-\tau \operatorname{sech}(2 \pi a / \omega) \cdot \tanh (2 \pi x / \omega)}{\sqrt{\tanh ^{2}(2 \pi a / \omega)-\tanh ^{2}(2 \pi x / \omega)}} \cdot i .
\end{aligned}
$$

Substituting (13) and (15) into boundary conditions (4), the unique solution can be derived as follows:

$$
a_{1}=0, \quad b_{1}=\frac{1}{\beta Q_{44}} .
$$

Therefore, by substituting (14) and (16) into (11), we can obtain the real analytic solution $w$, which meets the governing equation (3) and the boundary conditions (4).

\section{Stress Intensity Factor}

According to the distribution of cracks and the loadings of orthotropic composite plate, we select the stress intensity factor as follows [13]:

$$
K_{\mathrm{III}}=\lim _{z_{1} \rightarrow a+n \omega i}\left[2 \pi\left(z_{1}-(a+n \omega i)\right)\right]^{1 / 2} U_{1}\left(z_{1}\right) .
$$

Substituting (14) into (17), we obtain

$$
K_{\mathrm{III}}=\tau \sqrt{\frac{\omega \cdot \tanh (2 \pi a / \omega)}{2}}=Y \tau \sqrt{\pi a}
$$

where $Y=\sqrt{(\omega / 2 \pi a) \cdot \tanh (2 \pi a / \omega)}$ is called the shape factor and the stress intensity factor depends on $Y$. Labeling $K_{\text {III }}^{\prime}=$ $\tau \sqrt{\pi a}, K_{\mathrm{III}}^{\prime}$ is the stress intensity factor of a single central crack.

Suppose $\tau=1$ and $a=2$. As seen in Figure 2, the stress intensity factor $K_{\mathrm{III}}$ and the shape factor $Y$ increase rapidly with the increase in the distance between cracks and then reach a steady state. When $\omega \rightarrow \infty, Y=$ $\sqrt{(\omega / 2 \pi a) \cdot \tanh (2 \pi a / \omega)} \rightarrow 1$ and $K_{\mathrm{III}} \rightarrow K_{\mathrm{III}}^{\prime}$. In other words, when $\omega \rightarrow \infty$, the antiplane problem of periodic parallel cracks turns into the antiplane problem of a single central crack, and it is entirely consistent with the previous results.

\section{Stress Field and Displacement Field Near Crack Tip}

According to (17), in the vicinity of the periodic parallel cracks tip, we can obtain [11]

$$
\begin{gathered}
U_{1}\left(z_{1}\right)=\frac{K_{\mathrm{III}}}{\sqrt{2 \pi\left(z_{1}-(a+n \omega i)\right)}}, \\
(n=0, \pm 1, \pm 2, \ldots), \quad \text { as } z_{1} \longrightarrow a+n \omega i .
\end{gathered}
$$

Let

$$
z_{1}-(a+n \omega i)=r\left(\cos \theta+s_{1} \sin \theta\right) .
$$

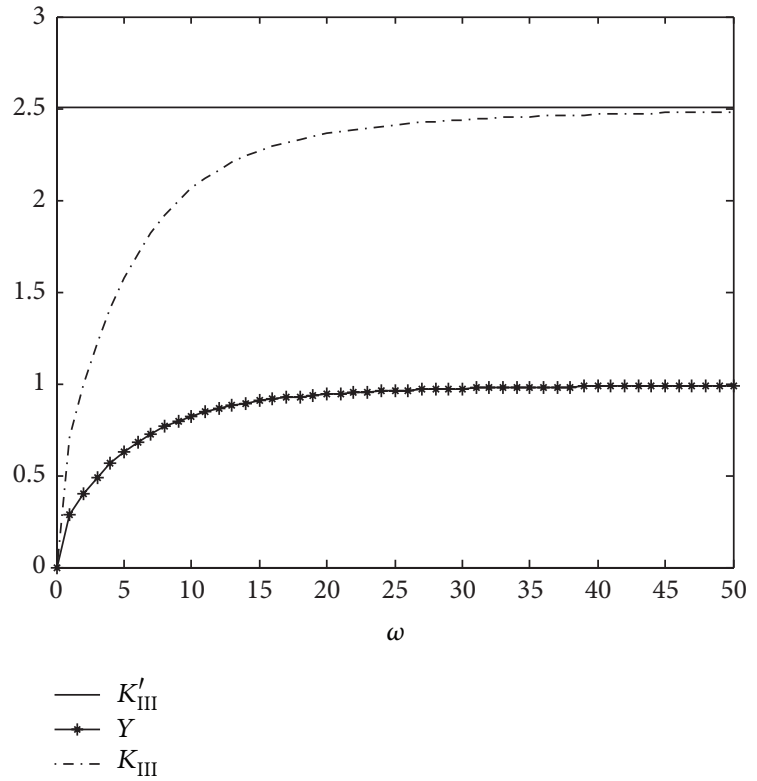

FIGURE 2: Variation curves of $K_{\mathrm{III}}^{\prime}, Y$, and $K_{\mathrm{III}}$ with crack spacing $\omega$.

So

$$
U_{1}\left(z_{1}\right)=\frac{K_{\mathrm{III}}}{\sqrt{2 \pi r\left(\cos \theta+s_{1} \sin \theta\right)}}, \quad \text { as } r \longrightarrow 0,
$$

where polar radius $r$ is the shortest distance to a point near the periodic parallel cracks tip.

Substituting (16) and (19) into (13), according to (1), we can obtain the analytic expressions for stress and displacement of the periodical parallel cracks tip:

$$
\begin{gathered}
\tau_{y z}=\frac{K_{\mathrm{III}}}{\sqrt{2 \pi}} \operatorname{Re}\left[\frac{1}{\sqrt{z_{1}-(a+n \omega i)}}\right], \\
\tau_{z x}=-\frac{K_{\mathrm{III}}}{\sqrt{2 \pi}} \operatorname{Re}\left[\frac{s_{1}}{\sqrt{z_{1}-(a+n \omega i)}}\right], \\
\omega=K_{\mathrm{III}} \sqrt{\frac{2}{\pi Q_{44} Q_{55}}} \operatorname{Im}\left[\sqrt{z_{1}-(a+n \omega i)}\right] .
\end{gathered}
$$

According to (20), (22) can be rewritten as follows:

$$
\begin{gathered}
\tau_{y z}=\frac{K_{\mathrm{III}}}{\sqrt{2 \pi r}} \operatorname{Re}\left[\frac{1}{\sqrt{\cos \theta+s_{1} \sin \theta}}\right], \\
\tau_{z x}=-\frac{K_{\mathrm{III}}}{\sqrt{2 \pi r}} \operatorname{Re}\left[\frac{s_{1}}{\sqrt{\cos \theta+s_{1} \sin \theta}}\right], \\
\omega=K_{\mathrm{III}} \sqrt{\frac{2 r}{\pi Q_{44} Q_{55}}} \operatorname{Im}\left[\sqrt{\cos \theta+s_{1} \sin \theta}\right] .
\end{gathered}
$$

\section{Conclusions}

In this paper, the antiplane problem of the periodic parallel cracks in an infinite linear elastic orthotropic composite 
plate is studied. The antiplane problem near periodic parallel cracks tip can be turned to find the solution of the boundary value problem of partial differential equation.

(1) The analytic expressions for stress intensity factor, stress, and displacement are obtained by constructing proper Westergaard stress function and using the complex variable function method and the boundary conditions.

(2) The stress intensity factor around the periodic parallel cracks tip depends on the shape factor $Y$.

(3) When $\omega \rightarrow \infty, Y \rightarrow 1$, and $K_{\mathrm{III}} \rightarrow K_{\mathrm{III}}^{\prime}$, it is concluded that the antiplane problem of the periodic parallel cracks degenerates into the antiplane problem of a single central crack.

\section{Conflict of Interests}

The authors declare no direct financial relation with any commercial entities mentioned in the paper that might lead to a conflict of interests.

\section{Acknowledgments}

This work was financially supported by the National Important Special Project of China (Grants nos. 2009ZX04001063063 and 2009ZX04001-051), National Hi-tech Research and Development Program of China (863, Grant no. 2008AA042503), The Natural Science Research Project of the Education Department of Henan Province (Grant no. 2011B460022), Zhengzhou Technology Innovation Team Project of China (Grant no. 10CXTD153), and Zhengzhou Science-Tech Tackle Key Project of China (Grant no. 10PTG400-3).

\section{References}

[1] F. Erdogan and M. Ozturk, "Periodic cracking of functionally graded coatings," International Journal of Engineering Science, vol. 33, no. 15, pp. 2179-2195, 1995.

[2] Y. Z. Chen and X. Y. Lin, "Collinear crack problem for a strip of functionally graded materials in anti-plane elasticity," Chinese Quarterly of Mechanics, vol. 27, no. 1, pp. 7-13, 2006.

[3] Y. Z. Chen, "Anti-plane problem of periodic crack in a strip of functionally graded materials," Acta Mechanica Sinica, vol. 36, no. 4, pp. 501-506, 2004.

[4] S. H. Ding and X. Li, "Anti-plane problem of periodic interface cracks in a functionally graded coating-substrate structure," International Journal of Fracture, vol. 153, no. 1, pp. 53-62, 2008.

[5] B. Wang and Y. Mai, "A periodic array of cracks in functionally graded materials subjected to transient loading," International Journal of Engineering Science, vol. 44, no. 5-6, pp. 351-364, 2006.

[6] Y. E. Pak and E. Goloubeva, "Electroelastic properties of cracked piezoelectric materials under longitudinal shear," Mechanics of Materials, vol. 24, no. 4, pp. 287-303, 1996.

[7] Z. H. Tong, C. P. Jiang, S. H. Lo, and Y. K. Cheung, "A closed form solution to the antiplane problem of doubly periodic cracks of unequal size in piezoelectric materials," Mechanics of Materials, vol. 38, no. 4, pp. 269-286, 2006.

[8] T. H. Hao, "An exact solution of the anti-plane parallel periodical transverse crack field in a bimaterial infinite plane," International Journal of Fracture, vol. 47, no. 3, pp. R49-R51, 1991.

[9] T. H. Hao and Y. C. Wu, "Elastic plane problem of collinear periodical rigid lines," Engineering Fracture Mechanics, vol. 33, no. 6, pp. 979-981, 1989.

[10] S. G. Lekhnitskii, Theory of Elasticity of an Anisotropic Elastic Body, Science Press, Beijing, China, 1963.

[11] W. Y. Yang, J. L. Li, and X. X. Zhang, Method of a Complex Variable for Fracture in Composite Materials, Science Press, Beijing, China, 2005.

[12] X. X. Zhang, C. Li, X. C. Cui, and W. B. Zhao, "Analysis of mode III collinear periodic cracks-tip stress field of an infinite orthotropic plate," Advanced Materials Research, vol. 446-449, pp. 2080-2084, 2012.

[13] W. B. Zhao, L. J Yu, X. X. Zhang, H. L. Xie, and Z. X. Hu, "Analysis of mode I periodic parallel cracks-tip stress field in an infinite orthotropic plate," Mathematical Problems in Engineering, vol. 2013, Article ID 412172, 8 pages, 2013. 


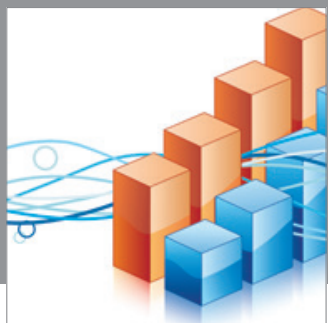

Advances in

Operations Research

mansans

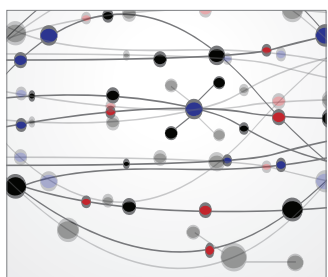

The Scientific World Journal
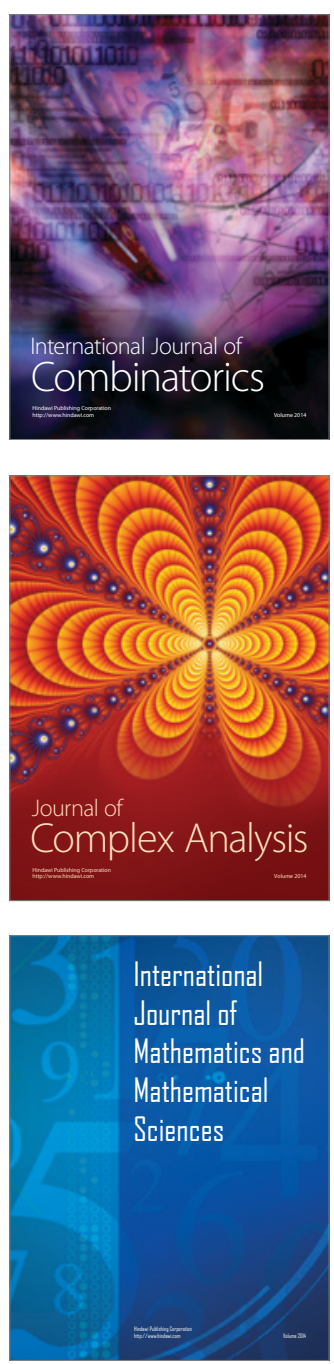
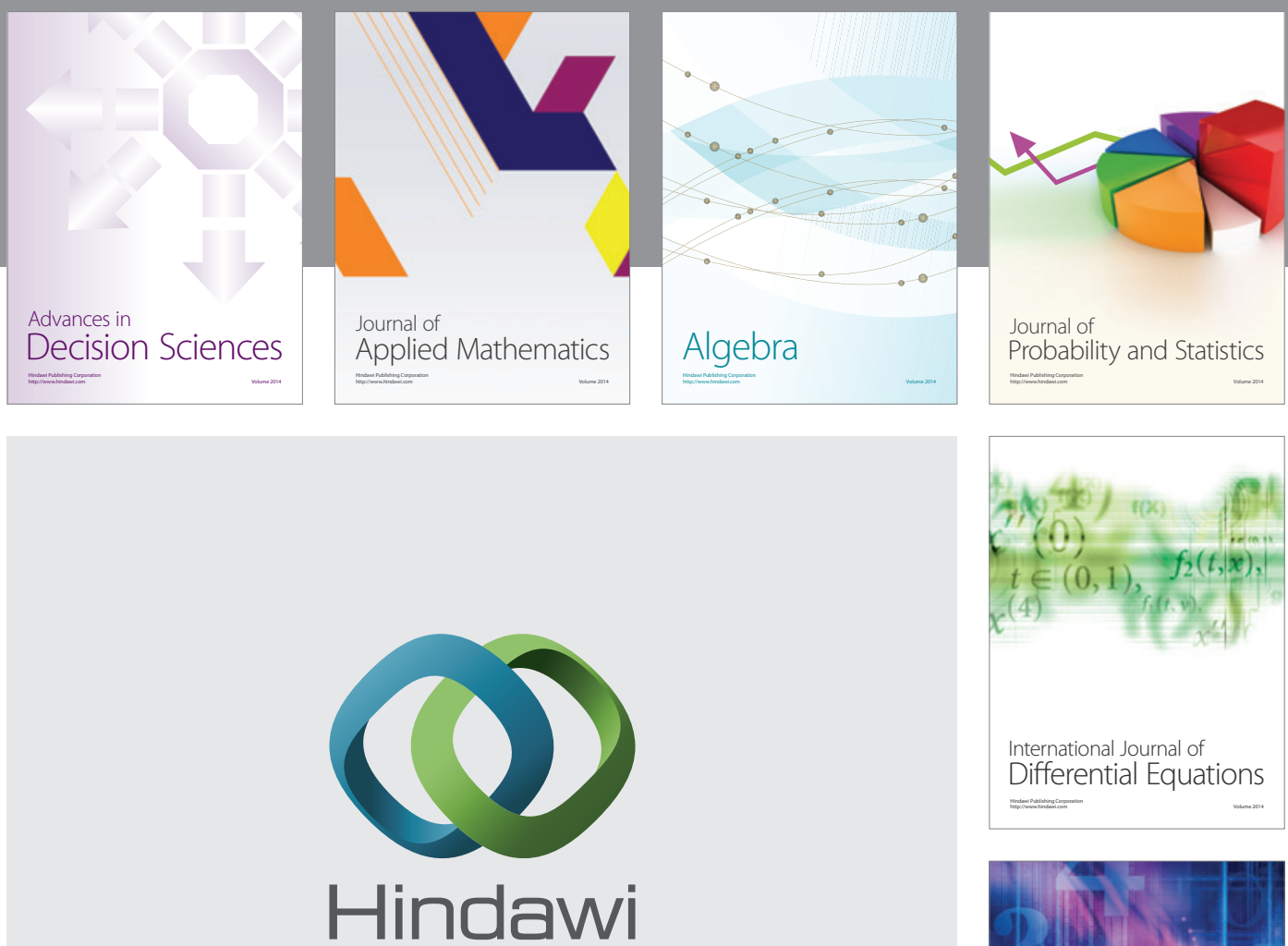

Submit your manuscripts at http://www.hindawi.com
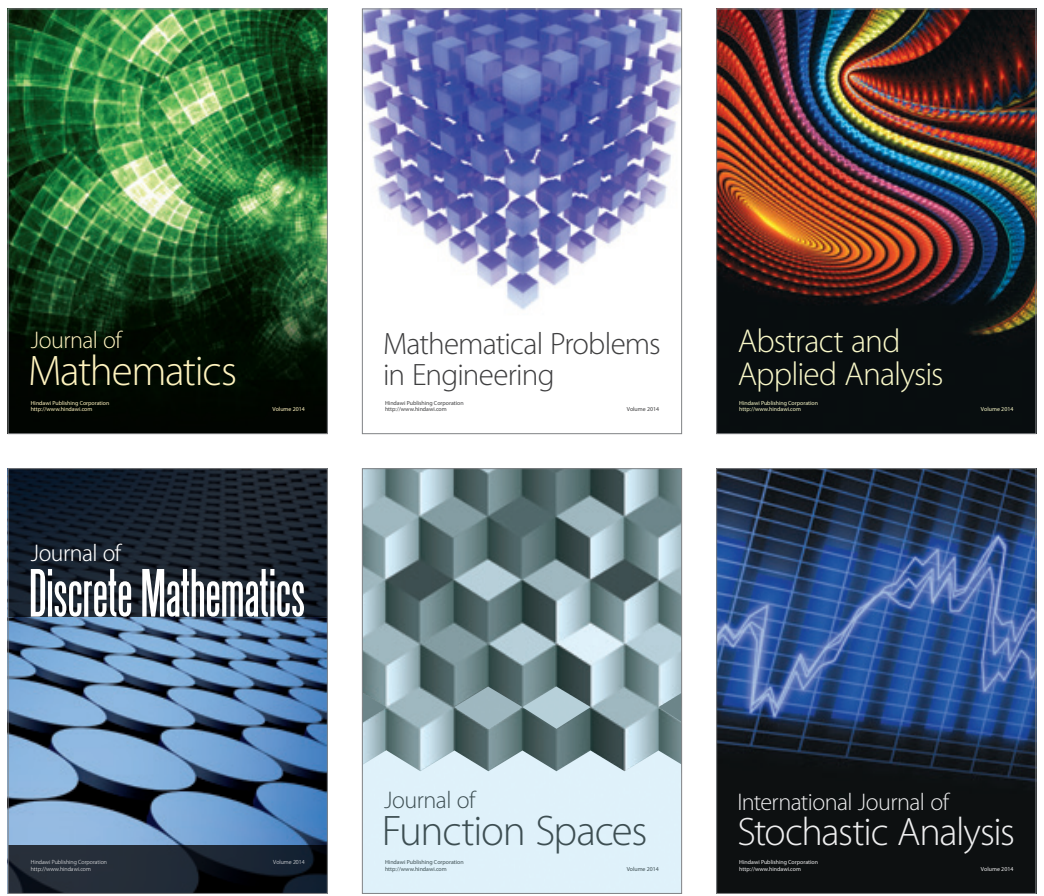

Journal of

Function Spaces

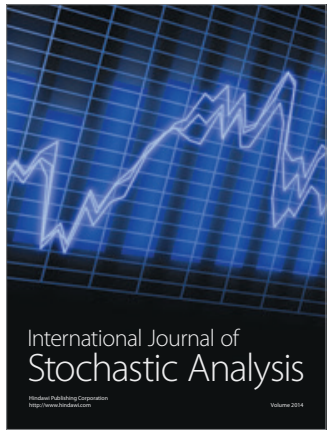

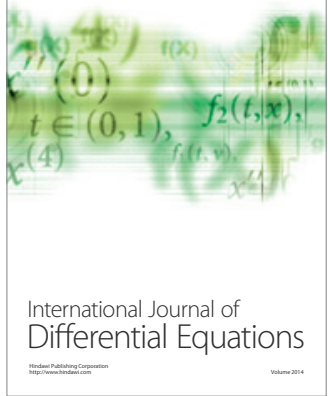
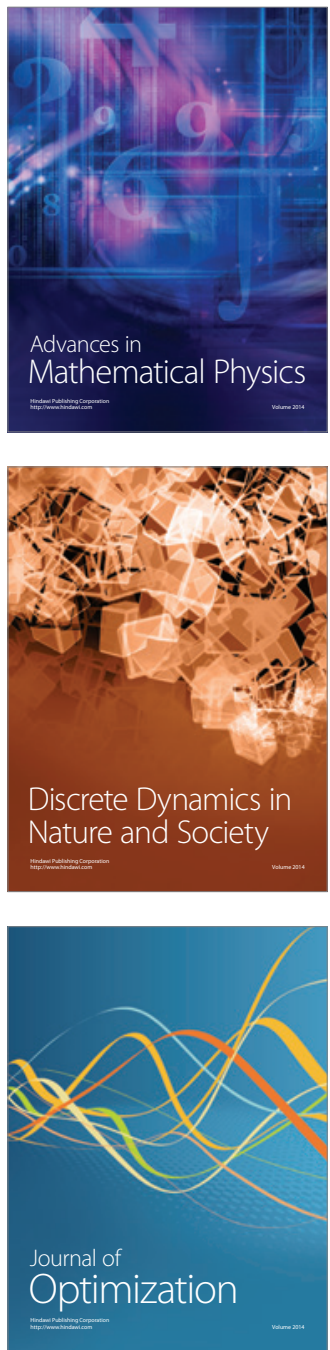